\title{
Technical Feasibility Does Not Guarantee Clinical Improvement: A Word of Caution for Valve-in-Valve Procedures in Small Surgical Prosthesis
}

\author{
Marie-Elisabeth Stelzmueller ${ }^{1}$, Bruno Mora $^{2}$, Guenther Laufer ${ }^{1}$ and Wilfried Wisser ${ }^{1 *}$ \\ ${ }^{1}$ Department of Cardiac Surgery, Medical University Vienna, Austria \\ ${ }^{2}$ Department of Cardiothoracic Anesthesia and Intensive Care, Medical University Vienna, Austria
}

*Corresponding author: Dr. Wilfried Wisser, Prof. Department of Cardiac Surgery, Medical University Vienna, Spitalgasse 23, A-1090 Vienna, Austria, Tel: 0043/1/404005620

\begin{abstract}
Background: The reoperation of old and frail patients with small tissue valves is a still challenging topic in cardiac surgery.

Case summary: An 85-years-old patient who underwent an aortic valve replacement (AVR) with a Mitroflow $21 \mathrm{~mm}$ and single coronary bypass operation 6 years ago, presented again dyspnea NYHA II-III. The echo showed a restenosis of the tissue valve and a mean gradient of $40 \mathrm{mmHg}$.

Because of age, frailty and calculated perioperative risk, the patient was evaluated for a transcatheter valve-in-valve implantation (ViV).

Due to the small inner diameter of the $21 \mathrm{~mm}$ Mitroflow, only a transfemoral (TF) $20 \mathrm{~mm}$ Edwards Sapien prosthesis was recommended. The TF-approach was impossible due to peripheral artery disease (PAD).

Therefore, we decided to perform an off-label procedure with an Edwards Sapien $20 \mathrm{~mm}$ TF-prosthesis through a transapical-(TA)-approach.

The transapical transcatheter aortic valve implantation (TA-TAVI) was performed successfully without any technical complication, but was damped by the fact, that the invasively measured peak-to-peak gradient was $21 \mathrm{mmHg}$ and echocardiography revealed a peak gradient of $16 \mathrm{mmHg}$.

Discussion: ViV in patients with small surgical tissue valves (SSTV) is technically feasible, but we have to pay attention to postoperative hemodynamic and clinical improvement.
\end{abstract}

\section{Introduction}

The reoperation of a degenerated SSTV is challeng- ing for standard cardiac surgery as well as for TAVI. Although it is technically feasible to perform a TAVI in a degenerated SSTV, we are still dealing with the problem of the high postoperative gradients.

Use of a SSTV frequently ends up in postoperative higher gradients, due to the small inner diameter and consecutively smaller aortic valve area. Additionally, there are significant varieties between the different companies (Table 1 ).

This case report points out the problem of TAVI in degenerated SSTV.

\section{Case Description}

Six years after surgical AVR with a $21 \mathrm{~mm}$ Mitroflow bioprosthesis and concomitant bypass surgery an 85-years-old female patient developed a restenosis of the aortic valve prosthesis. The AVPmean was $40 \mathrm{mmHg}$ and AVVmax was $4.4 \mathrm{~m} / \mathrm{s}$ with preserved left ventricular function.

Because of the age, frailty and calculated perioperative risk of $38.7 \%$ logistic EuroScore and 15\% EuroScore II the patient was evaluated for a ViV.

The inner diameter of the Mitroflow is $17 \mathrm{~mm}$, therefore a $20 \mathrm{~mm}$ Edwards Sapien Valve is suggested in a TF-approach.

Due to small iliac vessel diameter and PAD a TF-approach was impossible in this patient. A transaortic ap-

Citation: Stelzmueller ME, Mora B, Laufer G, Wisser W (2021) Technical Feasibility Does Not Guarantee Clinical Improvement: A Word of Caution for Valve-in-Valve Procedures in Small Surgical Prosthesis. Int Arch Cardiovasc Dis 5:038. doi.org/10.23937/2643-3966/1710038

Accepted: March 11, 2021: Published: March 13, 2021

Copyright: (C) 2021 Stelzmueller ME, et al. This is an open-access article distributed under the terms of the Creative Commons Attribution License, which permits unrestricted use, distribution, and reproduction in any medium, provided the original author and source are credited. 
Table 1: Significant varieties of SSTV between different companies.

\begin{tabular}{|l|c|c|c|}
\hline \multicolumn{1}{|c|}{ Aortic bioprosthesis } & $\begin{array}{c}\text { Labelled size } \\
(\mathbf{m m})\end{array}$ & $\begin{array}{c}\text { Inner diameter } \\
(\mathbf{m m})\end{array}$ & $\begin{array}{c}\text { External diameter/incl. sewing ring } \\
\text { (mm) }\end{array}$ \\
\hline Edwards Magna Ease & 19 & 18 & 24 \\
\hline Edwards Magna & 19 & 18 & 24 \\
\hline Edwards Perimount & 19 & 17.5 & 25 \\
\hline Medtronic Mosaic & 19 & 17.5 & 24 \\
\hline Medtronic Mosaic Ultra & 19 & 15.4 & 21 \\
\hline Sorin Mitroflow & 19 & 19 & 26 \\
\hline SJM Epic Supra & 19 & 20 & 26 \\
\hline Edwards Magna Ease & 21 & 20 & 28 \\
\hline Edwards Magna & 21 & 20 & 27 \\
\hline Edwards Perimount & 21 & 18.5 & 26 \\
\hline Medtronic Mosaic/Hancockll & 21 & 18.5 & 23 \\
\hline Medtronic Mosaic Ultra / Hancockll Ultra & 21 & 17.3 & 25 \\
\hline Sorin Mitroflow & 21 & 19 & 28 \\
\hline SJM Epic Biocor & 21 & 21 & 26 \\
\hline SJM Epic Biocor Supra & 21 & & 26 \\
\hline
\end{tabular}

proach would be beneficial for the patient due to the redo situation and the prior CABG, with two venous grafts anastomosed to the ascending aorta. A transsubclavian approach was not possible due to the small diameter of the subclavian vessel. Therefore, we decided to perform an off-label implantation of a TF-20 mm Sapien prosthesis through a TA-approach.

The setting was similar to usual TA-TAVI, except that use of the Sapien $20 \mathrm{~mm}$ TF-device for TA-approach.

The procedure was performed in general anesthesia in the operation room with the use of a C-arm. The TFsheath was introduced into the left ventricle through the apex. After pre-ballooning, the prosthesis was implanted, attended by angiography.

The TAVI was performed successfully without any technical complication. However, the excellent placement of the valve prosthesis with no paravalvular leak, was damped by the fact that the invasive measured peak to peak gradient was $21 \mathrm{mmHg}$ and echocardiography revealed a peak gradient of $16 \mathrm{mmHg}$.

The patient was extubated within the first postoperative day and the postoperative course was uneventful.

The follow up on echocardiography is shown in Table 2.

\section{Conclusion}

AVR in patients with small aortic roots or annuli is challenging, especially for inexperienced surgeons. To avoid a more complex aortic root annuloplasty to enlarge the native aortic annulus, many surgeons tend to rather implant smaller aortic valves. By offering SSTV with promising gradients, the industry was providing a comfortable and satisfying solution. Therefore, root enlargement surgery plays a minor role.
Table 2: Echocardiography Follow up.

\begin{tabular}{|l|l|l|l|}
\hline & Pre-operative & Discharge & 1 YFU \\
\hline AV Vmax & $4.4 \mathrm{~m} / \mathrm{s}$ & $3.8 \mathrm{~m} / \mathrm{s}$ & $3.3 \mathrm{~m} / \mathrm{s}$ \\
\hline AV Pmean & $40 \mathrm{mmHg}$ & $37 \mathrm{mmHg}$ & $27 \mathrm{mmHg}$ \\
\hline AV Ppeak & $70 \mathrm{mmHg}$ & $57 \mathrm{mmHg}$ & $45 \mathrm{mmHg}$ \\
\hline LVEF & $55 \%$ & $55 \%$ & $45 \%$ \\
\hline NYHA & II-III & II & II-III \\
\hline
\end{tabular}

Due to the promising developments and longer durability of bioprosthesis, they were used more generously even in younger patients.

However, life expectancy is increasing and we are going to face a growing number of patients with degenerated SSTV.

With the implantation of a SSTV, several problems have to be addressed.

There might be a, at least moderate, patient-prosthesis-mismatch (PPM) immediately after surgery, regarding the increasing number of obese patients with small native annulus size and rather big body surface area (BSA).

Additionally, if the tissue valve shows signs of degeneration a ViV might be feasible, but ending up with again a smaller aortic valve area and a higher postoperative gradient with missing clinical or haemodynamic improvement.

Regarding the presented case, the patient had a BMI of 27 and a BSA of $1.97 \mathrm{~m}^{2}$, requiring a Mitroflow valve size of $25 \mathrm{~mm}$ to avoid a PPM. So, with receiving a 21 $\mathrm{mm}$ Mitroflow the patient already had a moderate PPM after the first operation. With the ViV therefore it was unlikely to achieve an improvement of the symptoms. 
In literature a couple of novel approaches have been described to overcome these problems interventionalIy: Recent case series reported experience with a CoreValve placed supra-annular in the bioprosthesis with a serious increasing risk of coronary obstruction [1].

Other studies describe a high pressure ballooning of the SSTV with consecutive cracking of the prosthesis ring, but these procedures are still experimental bail out procedures with small caseload and have no evidence in larger clinical trials $[2,3]$.

However, in reflection of this case we should not end up with more technical experiments but come back to our surgical roots. We have to avoid the implantation of SSTV, especially in younger patients, by all means.

\section{References}

1. Adamo M, Fiorina C, Curello S, Chizzola G, Pezzotti E, et al. (2017) Self-expanding transcatheter aortic valve implantation for degenerated small Mitroflow bioprosthesis: Early and midterm outcomes. Eurolntervention 13: e1032-e1039.

2. Nielsen-Kudsk JE, Andersen A, Therkelsen CJ, Christensen EH, Jensen KT, et al. (2017) High-pressure balloon fracturing of small dysfunctional Mitroflow bioprostheses facilitates transcatheter aortic valve-in-valve implantation. Eurolntervention 13: e1020-e1025.

3. Johansen $\mathrm{P}$, Engholt $\mathrm{H}$, Tang M, Nybo RF, Rasmussen PD, et al. (2017) Fracturing mechanics before valve-in-valve therapy of small aortic bioprosthetic heart valves. Eurolntervention 13: e1026-e1031. 